\title{
Properties in Fe-Doped ZnS Thin Films
}

\author{
Jafarli Rufat*
}

Baku State University, Russia

ISSN: 2576-8840

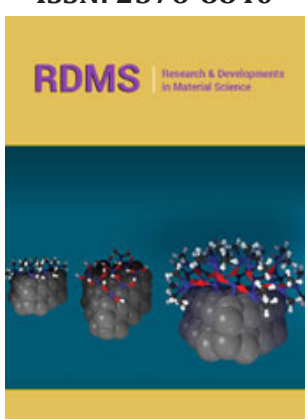

*Corresponding author: Jafarli Rufat, Baku State University, Russia

Submission: 眥 November 08, 2021

Published: 眥 November 16, 2021

Volume 16 - Issue 1

How to cite this article: Jafarli Rufat Properties in Fe-Doped ZnS Thin Films. Res Dev Material Sci. 16(1). RDMS.000879. 2021.

DOI: 10.31031/RDMS.2021.16.000879

Copyright@ Jafarli Rufat. This article is distributed under the terms of the Creative Commons Attribution 4.0 International License, which permits unrestricted use and redistribution provided that the original author and source are credited.

\begin{abstract}
Fe-doped ZnS single-phase thin films showing ferromagnetism have been successfully prepared by Chemical Bath deposition (CBD) on GaAs substrates. Field and temperature dependent magnetization curve indicate that the sample with a Curie temperature $T c$ as high as $270 \mathrm{~K}$. The X-ray diffraction and Atomic Force Microscopy (AFM) reveal that the thin films are well crystallized, Fe ions are substituted for $\mathrm{Zn}$ ions in the ZnS matrix and no trace of secondary phases or Fe clusters is detected. The experimental results are explained theoretically by spin-polarized density functional calculations within GeneralizedGradient Approximations (GGA), which indicates the observed high Tc could be mainly ascribed to the p-d exchange coupling between Fe ions and host elements.
\end{abstract}

Keywords: Fe-doped ZnS; First principles calculation; Ferromagnetic properties

\section{Introduction}

Transition-Metal (TM) doped wide gap II-VI semiconductors materials, such as ZnO, GaN and $\mathrm{ZnS}$, have been extensively investigated for potential magnetoelectronic or magnetooptical applications with the purpose of realizing of a high Curie temperature (Tc) [1-3]. Such TM-doped semiconductors called "Dilute Magnetic Semiconductor" (DMS) could be integrated with conventional semiconductor devices for spintronic applications [4-6]. In past years, many works have studied the doping properties of ZnS. A band structure model, based on the $p$ - $d$ and $d$ - $d$ level repulsions between the TM ions and host elements, has been successfully used to explain the magnetic ordering observed in all Mn-doped III-V and II-VI semiconductors. In this model, the carriers (electrons or holes) play an important role in stabilizing the ferromagnetism of DMS. In order to confirm above model, we also perform firstprinciples calculations based on the local spin density approximations to study the magnetic properties of $\mathrm{ZnS}: \mathrm{Fe}$ system. In this work, the $\mathrm{Zn}_{\mathrm{x}} \mathrm{Fe}_{1-\mathrm{x}} \mathrm{S}$ thin films are prepared by lowpressure Metalorganic Chemical Vapor Deposition (MOCVD) equipment on Si substrates [7]. Structural and morphological studies are done by means of X-ray diffraction (XRD) technique using $\mathrm{Cu}$ K $\alpha$ irradiation on an 800W Philips 1830 powder diffractometer. X-ray Photoelectron Spectroscopy (XPS) measurement is carried out on VGESCALAB MK II instrument where Mg $\mathrm{K} \alpha \mathrm{X}$-ray $(\mathrm{h} v=1253.6 \mathrm{eV})$ is used as the emission source. The magnetization measurements are performed by a Quantum Design superconducting quantum interference device (SQUID) system [1-6].

\section{Experiments and Theory}

ZnS:Fe single-crystal thin films are grown on Si substrates by low-pressure CBD at substrate temperature of $350{ }^{\circ} \mathrm{C}$. The substrates are cleaned by ultrasonic with a sequence of trichloroethylene, acetone, ethanol and deionized (DI) water, and etched in the mixture solution of a $\mathrm{H}_{2} \mathrm{SO}_{4}: \mathrm{H}_{2} \mathrm{O}_{2}: \mathrm{H}_{2} \mathrm{O}=3: 1: 1$ at room temperature for $10 \mathrm{~min}$. Before put into the growth chamber, the chemical etched substrates are rinsed in DI water again and finally dried by nitrogen blowing. Precursors consist of ironpentacarbonyl $\left(\mathrm{FeCl}_{3}\right)$, dimethylzinc (DMZn) and $\mathrm{H}_{2} \mathrm{~S}$. In our experiment, the growth pressure fixed is 76 Torr using a homemade 
horizontal reactor, and high purity hydrogen is used to carry the organometallics into the reaction chamber with the total gas flow rate of $21 \mathrm{~min}^{-1}$. The Si substrate is cleaned in hydrogen ambient at $600{ }^{\circ} \mathrm{C} 10 \mathrm{~min}$, which is demonstrated to be an effective method to remove the oxide layer and residual surface contaminants. Films are grown for $30 \mathrm{~min}$ and the film thicknesses are about $200 \mathrm{~nm}$. The Fe content in the reactor is controlled by varying the $\mathrm{H}_{2}$ flow rate. The resulting $\mathrm{Fe}$ concentration in the sample is determined using XPS. The structure of the ZnS:Fe thin films is characterized by X-ray diffractometers. Surface morphology of the films is investigated by AFM operated in tapping mode. The hightemperature $(100 \mathrm{~K})$ magnetic properties have been assessed using a SQUID magnetometer.

The calculations are performed using VASP code, based on the spin density functional theory. For the exchange and correlation potential, the generalized gradient approximation (GGA) is used. All plane waves with a cutoff energy of $300 \mathrm{eV}$ are used in the basis function. For all the Fe-doped systems, a 64 atoms supercell is used for the defect calculations. The lattice constants of the supercells are kept fixed to that of pure $\mathrm{ZnS}$.

\section{Results and Discussion}

The structure of $\mathrm{ZnS}$ :Fe thin film is examined by $\theta-2 \theta$ X-ray diffraction system using $C u K \alpha$ radiation in the range $20^{\circ}-80^{\circ}$, which is shown in Figure 1. Firstly, strong ZnS:Fe (200) and (400) alloy peaks are observed at $2 \theta=33.01^{\circ}$ and $2 \theta=69.30^{\circ}$. Si substrates (200) and (400) peak are apparent near $2 \theta=31.61^{\circ}$ and $2 \theta=66.04^{\circ}$, respectively. No other peaks above the noise of the background are detected. From the Figure 1 rocking curve of $\mathrm{ZnS}$ (200) diffraction peak, it is clearly that the diffraction peaks indicate that $\mathrm{ZnS}$ single crystal grown on GaAs (200) substrate with cubic structure. And the ZnS:Fe thin film is phase pure with a (200) curve FWHM of 0.34 degrees. It can be explained that Fe ions substitute partly $\mathrm{Zn}$ ions and lead to the lattice structure difference. Addition, the $\mathrm{X}$-ray diffraction results are comparable with those of ZnS films grown on $\mathrm{Al}_{2} \mathrm{O}_{3}$ sapphire substrates under the same conditions. It is obviously shown that $\mathrm{ZnS}$ single crystal grown on $\mathrm{Al}_{2} \mathrm{O}_{3}(006)$ substrate is hexagonal structure, but the sample grown on $\mathrm{Si}(200)$ is cubic structure. The $\mathrm{ZnS}$ structure relates to the substrate and the Fe content, and the lattice structure is gradually changes from hexagonal to cubic structure under different substrates.

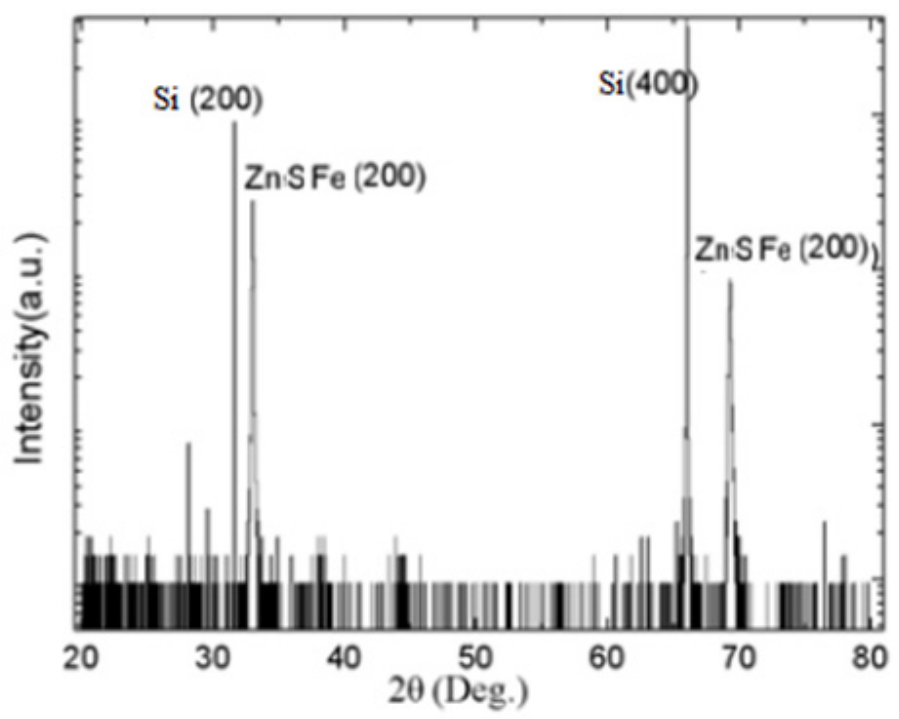

Figure 1: XRD patterns for the Fe-doped $\mathrm{ZnS}$ thin film on the Si substrates.

The fraction of Fe incorporated into the $\mathrm{ZnS}$ singlephase film is determined using X-ray photoelectron spectroscopy (XPS). Data of the XPS indicate the relative concentration of Fe to $\mathrm{Zn}$ to be $11 \%$ for this sample.

AFM is used to investigate the effect of Fe doping on the ZnS single crystal thin film morphology. Single phase films are typically non-specular and exhibit large values for the root mean squared roughness, which shows in Figure 2. The morphology of the multiphase films exhibits a much different morphology. A $2 \mathrm{~nm} \times 2 \mathrm{~nm}$ AFM micrograph for the Fe-doped $\mathrm{ZnS}$ thin films during the process of CBD is exhibited in Figure 2. The film thickness is $200 \mathrm{~nm}$ and no precipitates like $\mathrm{Fe}$ cluster. The temperature-dependent magnetization $(M-T)$ curve for single-phase Fe-doped ZnS sample are measured using a SQUID magnetometer with a magnetic field of 10000e, applied perpendicular to the film plane. Measurements are taken at the relatively large applied magnetic field in order to increase the magnetic signal from thin film with respect to the large diamagnetic response of the Si substrate. Measurements have also been taken at 30000e and shown similar temperature dependence. The results of magnetization subtract of the diamagnetic contribution of the GaAs substrate. The $d c$ zero-field-cooled (ZFC) and the field-cooled (FC) magnetization curves are shown in Figure 3. Measurements are performed from 5 to $300 \mathrm{~K}$. The FC is obtained by measuring the magnetic moment of the sample in a magnetic field of 1000 Oe during cooling. The ZFC measurement 
is obtained by first cooling the sample to $5 \mathrm{~K}$ in zero fields and then warming it in the same field as that of the FC measurement. The ZFC magnetization shows stronger temperature dependence than the FC one below 30K. ZFC and the FC magnetization curves are measured overlap after high temperature about $180 \mathrm{~K}$ with a paramagnetic behavior. This temperature below which the ZFC-FC curves separate, the ferromagnetic ordering sets are shown clearly in Figure 3 [2-4] displays a ferromagnetic behavior persisting up to $270 \mathrm{~K}$. Therefore, it obtains the Curie temperature $T c$ of $270 \mathrm{~K}$ in this system. As seen in Figure 3 the sample displays ferromagnetic ordering and a decrease with increasing of temperature. Moreover, ferromagnetic transition temperatures of previous report are focus in other material. For example, A transition temperature more than room temperature is obtained in Fe-doped in $\mathrm{ZnO}: \mathrm{Cu}$ reported by $\mathrm{S} J$ [5]. In the Co-doped ZnS samples, room-temperature magnetic hysteresis is observed [2]. But up to date, there is no work reporting Fe-doped ZnS thin film with high Tc of $270 \mathrm{~K}$ grown by MOCVD. Figure 4 displays the results of magnetization as a function of applied magnetic field measured for $\mathrm{ZnS}$ :Fe film at $100 \mathrm{~K}$. The total magnetization could be described as an algebraic sum of various contributions. The substrate diamagnetic contribution is subtracted from the total magnetization signal. The remaining data consist of the paramagnetic and ferromagnetic contributions from the $\mathrm{ZnS}$ :Fe thin film. The well-defined hysteresis loops show that the ZnxFe1-xS film are clearly ferromagnetic at $100 \mathrm{~K}$. At $100 \mathrm{~K}$, the saturation magnetization $(M S)$ is $1.82 \times 10^{-5} \mathrm{emu}$ with a remanence magnetization $(M R)$ of $0.31 \times 10^{-5} \mathrm{emu}$ and a coercive field $(H C)$ of 50 Oe. GaAs substrate and $\mathrm{ZnS}$ are diamagnetic, so the magnetization data indicate that the ferromagnetization in the present study is because Fe-doped in ZnS thin film and formed ZnxFe1-xS DMS [8].
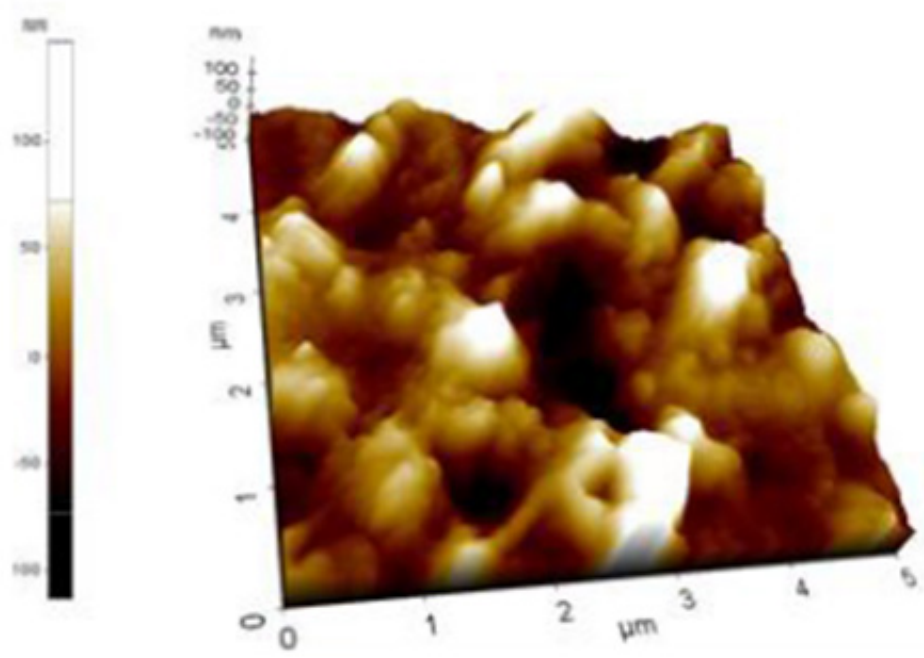

Figure 2: (color online)AFM image of the Fe-doped $\mathrm{ZnS}$ film on Si.

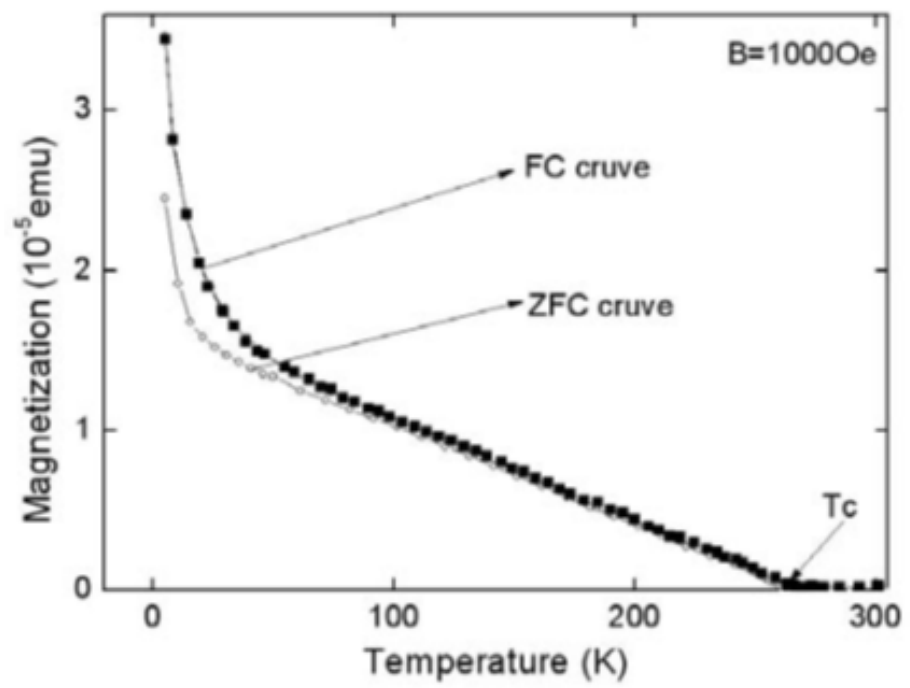

Figure 3: The FC-ZFC M-T curve of the Fe-doped ZnS shows temperature-dependent magnetization of both zero field - cooled (down - curve) and field - cooled (up-curve) in the field of 1.0KOe. 


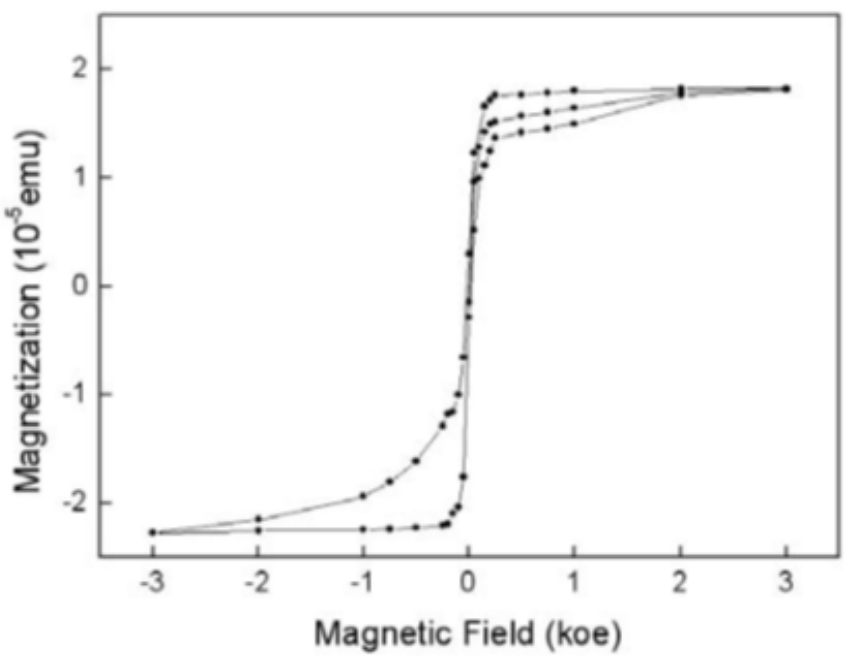

Figure 4: M-H curve of the sample taken at $100 \mathrm{~K}$ after the necessary background diamagnetic subtraction, the magnetic field used is from 0 up to $0.3 \mathrm{~T}$.

The origin of the ferromagnetism in DMS materials is still not clearly understood. To further demonstrate that the Fe-doped $\mathrm{ZnS}$ thin films are favorable for high temperature ferromagnetism, we model 64-atom cell to simulate the magnetic interactions of Fe atoms by performing firstprinciples spin-polarized Density Functional Theory (DFT) calculations within Generalized-Gradient Approximation (GGA). We can find that these magnetic interactions are mainly strong $p$ - $d$ coupling between the Fe $3 d$ states and S $2 p$ states. The calculated total energy differences between ferromagnetic (FM) and anti-ferromagnetic (AFM) configuration $(\triangle E=E F M-E A F M)$ are $46 \mathrm{meV}$, indicating that ferromagnetic ordering is favorable for $\mathrm{ZnS}: \mathrm{Fe}$ system. Therefore, the observed ferromagnetism should be the intrinsic behavior of Fe-doped $\mathrm{ZnS}$ thin film.

\section{Conclusion}

In summary, we have reported the ferromagnetic properties of single-phase ZnS:Fe thin films prepared on Si substrates by CBD as a new method. Field and temperature dependent magnetization curve indicated that a high curie temperature $T c=270 \mathrm{~K}$. The XRD and AFM used for investigating the structural and surface characterization, the results indicated that no Fe clusters and other compounds are formed in the samples. The experimentally observed high Tc could be mainly ascribed to the p-d exchange coupling between Fe ions and host elements.

\section{References}

1. Mirov S, Fedorov V, Moskalev I, Mirov M, Martyshkin D (2013) Frontiers of mid-infrared lasers based on transition metal doped II-VI semiconductors. J Lumin 133: 268-275.

2. Gloeckler M, Sankin I, Zhao Z (2013) CdTe solar cells at the threshold to 20\% efficiency. IEEE J Photovolt 3(4): 1389.

3. Farias PM, Santos BS, de Thomaz AA, Ferreira R, Menezes FD, et al. (2008) Fluorescent II- VI semiconductor quantum dots in living cells: nonlinear microspectroscopy in an optical tweezers system. J Phys Chem B 112(9): 2734-2737.

4. Ravikumar AP, De Jesus J, Tamargo MC, Gmachl CF (2015) High performance, room temperature, broadband II-VI quantum cascade detector. Appl Phys Lett 107(14): 141105.

5. Li J, Diercks DR, Ohno TR, Warren CW, Lonergan MC, et al. (2015) Sol Energy Mater Sol Cells 133: 208.

6. Gessert TA, Asher S, Johnston S, Young M, Dippo P, et al. (2007) Analysis of CdS/CdTe devices incorporating a ZnTe:Cu/Ti Contact. Thin Solid Films 515(15): 6103-6106.

7. Lee DU, Kim SP, Lee KS, Pak SW, Kim EK (2013) Band gap modulation of ZnTe1-xOx alloy film by control of oxygen gas flow rate during reactive magnetron sputtering. Appl Phys Lett 103: 263901.

8. Nakasu T, Kobayashi M, Togo H, Asahi T (2014) Oriented Domain Formation During Growth of ZnTe on m-Plane Sapphire by MBE. J Electron Mater 43: 921-925.

For possible submissions Click below: 